Article

\title{
Determining Appropriate Lane-Changing Spacing for Off-Ramp Areas of Urban Expressways
}

\author{
Zhufei Huang ${ }^{1}$, Zihan Zhang ${ }^{2}$, Haijian Li ${ }^{1} * * \mathbb{C}$, Lingqiao Qin ${ }^{3}$ and Jian Rong ${ }^{1}$ \\ 1 Beijing Engineering Research Center of Urban Transportation Operation Support, 100 Pingleyuan, \\ Chaoyang District, Beijing University of Technology, Beijing 100124, China; \\ huangzhufei@emails.bjut.edu.cn (Z.H.); jrong@bjut.edu.cn (J.R.) \\ 2 China Academy of Urban Planning \& Design, Beijing 100044, China; 18801072722@163.com \\ 3 TOPS Laboratory, Department of Civil and Environmental Engineering, University of Wisconsin-Madison, \\ 1415 Engineering Drive, Madison, WI 53706, USA; lingqiao.qin@gmail.com \\ * Correspondence: lihaijian@bjut.edu.cn
}

Received: 15 March 2019; Accepted: 28 March 2019; Published: 8 April 2019

check for updates

\begin{abstract}
Congestion has become a significant issue in recent years and has greatly affected the efficiency of urban traffic operation. Random and disorderly lane-changing behavior greatly reduces traffic capacity and safety. This paper is mainly concerned with the relationship of lane-changing spacing intervals provided by off-ramp facilities and traffic flow conditions. Through field investigations in Beijing, several typical lane-changing behaviors at off-ramp areas are analyzed. By using field traffic data and actual road geometry parameters, VISSIM-based micro-behavior simulations at off-ramp areas are implemented to obtain traffic flow conditions with different lane-changing spacing intervals and other model parameters, such as traffic volume and ratio of off-ramp vehicles. Then, the numerical relationships between traffic flow state and model parameters can be shown. The results show that with increasing traffic volume and the ratio of off-ramp vehicles, the lane-changing spacing interval required by vehicles should be increased. For the same ratio of off-ramp vehicles, if the traffic volume increases by $100 \mathrm{pcu} / \mathrm{h} / \mathrm{lane}(\mathrm{pcu}$ is a unit to stand for a standard passenger car), the corresponding lane-changing spacing interval should be increased by a spacing of 50-100 $\mathrm{m}$ to avoid increasing congestion. Based on the results of this paper, smart lane management can be implemented by optimizing lane-changing spacing intervals and lane-changing behaviors to improve traffic capacity.
\end{abstract}

Keywords: traffic engineering; traffic simulation; lane-changing behavior; off-ramp

\section{Introduction}

With the rapid development of society and the economy, cities are enlarged every year, especially in China. The quantity of motor vehicles continues to increase. In many large metropolitan areas, the road capacity cannot meet actual daily travel demands. As the main artery of urban traffic, urban expressways play a key role in daily urban transportation throughout the road network. The continual growth of daily travel demand leads to significant issues of traffic safety and congestion, which have seriously affected the efficiency of urban traffic operations. The Highway Safety Manual [1] shows that insufficient lane-changing spacing greatly increases the possibility of crashes, which could lead to safety problems and congestion. One of the main causes of traffic congestion is random and disorderly lane-changing behaviors. Bad driving behaviors will cause additional traffic delay, for example, at signalized road intersections, driving behavior has a serious impact on traffic delays [2]. Non-standard lane-changing behavior significantly influences road capacity of urban expressways. Lane-changing behaviors in off-ramp areas is a typical mandatory lane-changing behavior, which leads 
to remarkable influence on the following or adjacent traffic streams, especially for congestion flow. Therefore, determining appropriate lane-changing spacing intervals for off-ramp vehicles and guiding reasonable lane-changing activity are urgent problems for traffic researchers and engineers.

This paper is concerned with lane-changing spacing intervals and reasonable lane-changing locations in off-ramp areas of urban expressways. This paper searches for the optimizing mechanism of lane-changing spacing intervals to promote the road capacity of existing off-ramps by guiding and restraining driver lane-changing behaviors.

Based on the research in this paper, two ways can be realized to solve the off-ramp congestion in engineering by determining reasonable lane-changing spacing intervals for certain off-ramp areas and traffic volume demands. Findings can be applied in the following aspects: (1) to further optimize lane-changing behaviors to improve road capacity, (2) to provide a reference for location selection and design of urban expressway entrances and exits, and (3) to guide the design for appropriate combinations of strategy in lane-changing behavior.

The remainder of this paper is organized into the following sections: The next section details the analysis of lane-changing behaviors. Then, the model and simulation section gives the simulation scenarios and parameters. The next section presents the simulation results, analyses, and discussion. Finally, the concluding remarks and future work are presented.

\section{Literature Review}

\subsection{Impact of Lane-Changing Behavior}

Lane-changing behavior is becoming a significant concern in the research community. Many researchers have investigated this issue by proposing new or modified models [3-7], by simulating lane-changing behaviors in micro- or macro-scenarios [5,8-12], or by analyzing field data from traffic sensors $[3,4,13-16]$.

For mandatory lane changes, Cao et al. [3] presented an exploratory study of lane change execution and proposed a lane-changing behavior model on arterial roads. For off-ramp areas, Liu et al. [6] presented a second-order partial differential equation model that was used to simulate both compulsive lane-changing behavior and free lane-changing behavior.

By using field vehicle trajectory data, Jin [4] developed a multi-commodity, behavioral Lighthill-Whitham-Richards (LWR) model of lane-changing traffic flow and calibrated fundamental diagrams corresponding to a triangular car-following fundamental diagram with NGSIM data. Keyvan et al. proposed that [8,9] the decision process of lane-changing maneuvers was investigated by driving on a freeway with participants. The participants revealed that the choices to change lanes are related to their speed choice, and there are four basic strategies for lane-changing decisions: speed leading, speed leading with overtaking, lane leading, and traffic leading. Wang et al. [11] examined the impact of driver propensity on lane-changing. Driver propensity is her/his emotion state or the corresponding preference of a decision or action; the researchers considered dynamic recognition of a driver's propensity in simulation scenarios by analyzing their psycho-physic characteristics. In [14], Du et al. studied the relationship of high-occupancy vehicle facilities and lane-changing behaviors. They used lane change data with a high resolution in both time and space from videos of lane-changing frequency, comparing data with continuous-access, high-occupancy vehicle facilities and limited-access, high-occupancy vehicle facilities. Zeng et al. [17] found pertinent characteristics by analyzing lane-changing times with the total deviation velocity. Wang et al. [18] proposed parallel driving in cyber-physical-social space, considering interactions among vehicles, human drivers, and information.

\subsection{Impact of Weaving Sections}

For weaving sections, Mai et al. [19] investigated a lane-change distribution advisory application based on cooperative intelligent transport systems for weaving vehicles. They alleviated the 
lane-changing concentration problem by coordinating weaving vehicles. Tang et al. [20] studied the impacts of lane width and the number of lanes on multilane traffic flow. The researchers proposed a new multilane traffic flow model exploring the influences of lane width, closing lanes, and changing the number of lanes on traffic flow in each lane.

Because improper mandatory lane-changing maneuvers in the vicinity of off-ramps jeopardize traffic efficiency and safety [21], appropriate locations for advance warnings of mandatory lane changes will play a significant role in optimizing lane-changing behaviors for off-ramp vehicles. Seraj [22] proposed a model to consider both speed and density difference as lane-changing incentive and to study variable speed limit compliance impacts on lane-changing patterns. He found that voluntary compliance to variable speed limits had insignificant implications for compliance behavior, and variable speed limit compliance improvement will be necessary. This will eventually bring favorable change in collective lane-changing patterns. Yun et al. [23] studied in-vehicle navigation information impacts on lane-changing behavior at expressway diverge segments. They used a driving simulator to compare data of lane-changing position and merging gap from field surveys and simulations. Their results showed that traffic flow density and the time point of the initial navigation will vary lane-changing behavior. Under medium to high density conditions, in-vehicle navigation information will have a significant, positive impact on lane-changing safety. They also suggested that it will be better if in-vehicle navigation information can be provided earlier, within range of $2 \mathrm{~km}$ upstream of the off-ramp. This research demonstrated that appropriate out-vehicle guiding information and in-vehicle navigation information will help drivers achieve reasonable and safe lane-changing behaviors. However, determining appropriate lane-changing spacing intervals and reasonable lane-changing locations will be the primary concern under different traffic conditions. As Wang et al. [11] noted, psycho-physical characteristics of drivers will influence their lane-changing behaviors. The secondary concern will be how to guide and restrain driver behavior using internal vehicle information (i.e., navigation, head-up display) and external vehicle information (i.e., traffic signs, markings, and variable message sign).

For merging/diverging behavior on freeways, Marczak et al. [24] compared and analyzed two empirical trajectory datasets, and then found the acceptable gaps and rejected gaps in the conceptual model of merging behavior. Leclercq et al. [25] calibrated and analyzed the relaxation model with only one parameter E, and utilized NGSIM trajectory data for calibration and verification. Knoop et al. [26] studied the impact of the on-ramp and merger rates of changing in lane distribution as a result of variable speed limit. Laval and Daganzo [27] used a model to track lane converters accurately and obtained four measurement parameters. Zheng [28] discussed the methods and characteristics of the lane-changing decision-making process model and the quantitative lane-changing behavior impact model on surrounding lanes.

\section{Lane-Changing Behavior Analysis of Off-Ramp Areas}

At off-ramp areas, drivers often change lanes subconsciously. Because of their own driving habits and the road conditions, the behaviors of some drivers are disorderly and non-standard. Vehicles, according to their destination at the off-ramp area, can be divided into two categories: mainline vehicles and off-ramp vehicles. The lane-changing behaviors of these mainline and off-ramp vehicles will be analyzed in this section.

\subsection{Lane-Changing Behavior of Through Vehicles}

For single lane conditions, the mainline and off-ramp vehicles will present a simple diverging flow phenomenon. In fact, at the off-ramp area of urban expressways, most off-ramps are multiple lanes. Ideally, all of the vehicles have finished their lane-changing behaviors when they approach off-ramp areas. The mainline vehicles have driven to the straight lane, and the off-ramp vehicles have driven to the outer lane before they get to the off-ramp area. Then, these vehicles present a simple diverging flow at the off-ramp area. 
Figure 1 shows the actual lane-changing behaviors of multi-lane off-ramps under different road conditions. The first condition is a no traffic jam condition, as shown in Figure 1a. As different traffic speeds are in different lanes, some through vehicles will change lanes at the driver's own will and drive to the faster or more comfortable lane to improve their own driving experience. The second condition is a traffic jam condition where traffic volume of through vehicles is too large at the off-ramp area. All of the mainline vehicles move slowly, but the off-ramp vehicles are not that congested. Consequently, some of the main-line vehicles choose to change lanes to the off-ramp lane illegally and then drive to the straight lane before the exit, as shown in Figure 1b. The third condition occurs at the off-ramp area, where the road is congested by off-ramp saturation or over-saturation. All of the off-ramp vehicles move slowly, and then some of the off-ramp vehicles take up the straight lane to finish their lane-changing behavior. This condition will extrude the spacing of through vehicles, which makes some of the main-line vehicles change lanes to the inner lane. It will make the traffic flow worse, as shown in Figure 1c.

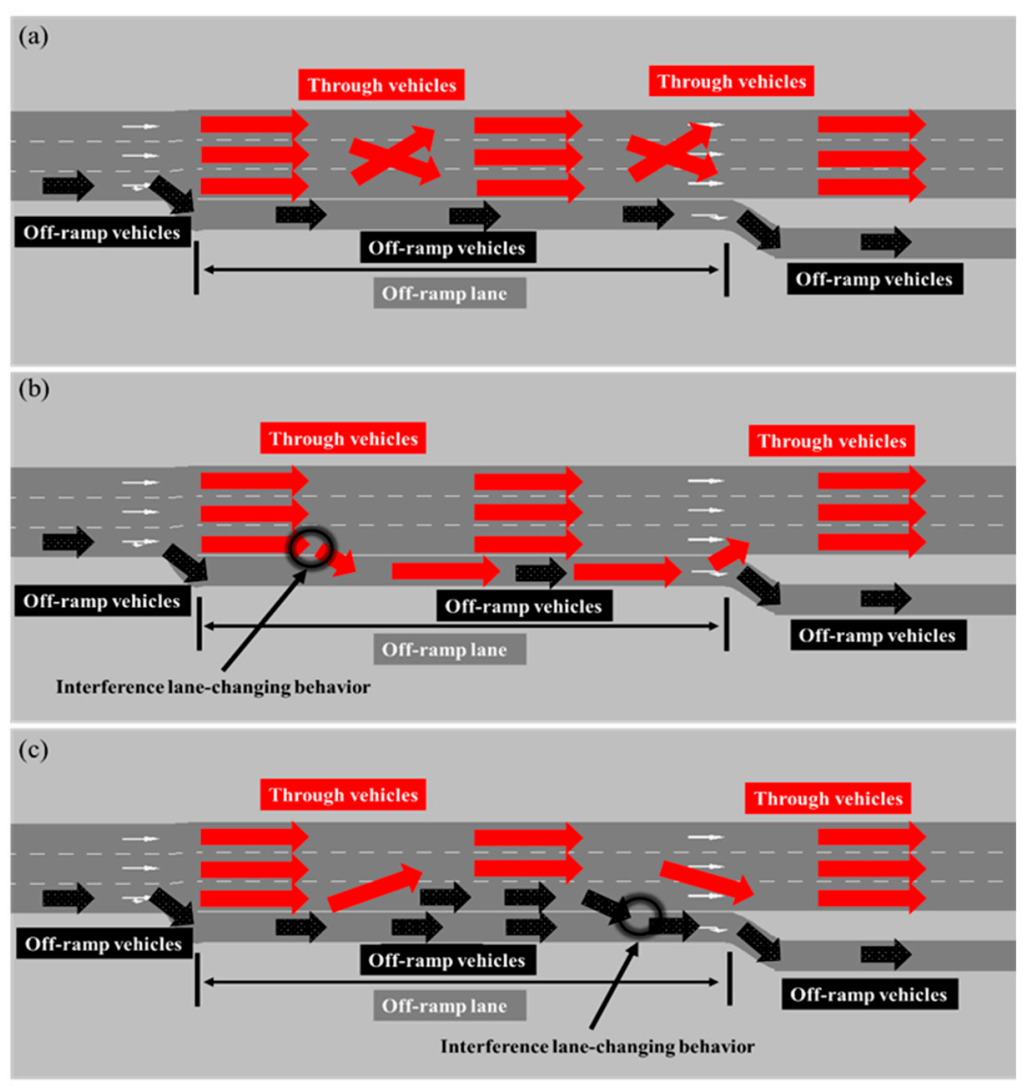

Figure 1. Lane-changing behaviors of mainline through vehicles under different traffic conditions: (a) no traffic jam; (b) through traffic flow saturation or over-saturation; and (c) off-ramp traffic flow saturation or over-saturation.

\subsection{Lane-Changing Behavior of Off-Ramp Vehicles}

Under non-standard driving conditions, the off-ramp vehicles often have the kind of driving behavior shown in Figure 2. The first condition is shown in Figure 2a. Under no traffic jam conditions, due to less familiarity of the road environment or to bad driving habits, some of the off-ramp vehicles do not finish their lane-changing behavior from the inner lane to outer lane. The off-ramp vehicles conflict with the mainline vehicles, which cause rear vehicle delay. The second condition occurs when the straight lanes are saturated, the vehicles at the off-ramp area move slowly. The drivers of the off-ramp vehicles will drive to the outer lane for exiting as early as possible when they realize the road is congested ahead. Some off-ramp vehicles will continue to drive in the inner lanes, but it will be 
difficult to finish their lane-changing behavior to the ramp on time. Consequently, their lane-changing behaviors will aggravate the traffic congestion at the off-ramp area, as shown in Figure $2 b$. The third condition is shown in Figure 2c. The off-ramp lanes are saturated or over-saturated. In this situation, some off-ramp vehicles will comply with the provisions of the road for exiting the ramp, and there will be some who choose to change lanes to the straight lane for cutting in line. To achieve exiting the ramp, their behaviors will aggravate the congestion of the road and cause congestion of the through lanes at some point.
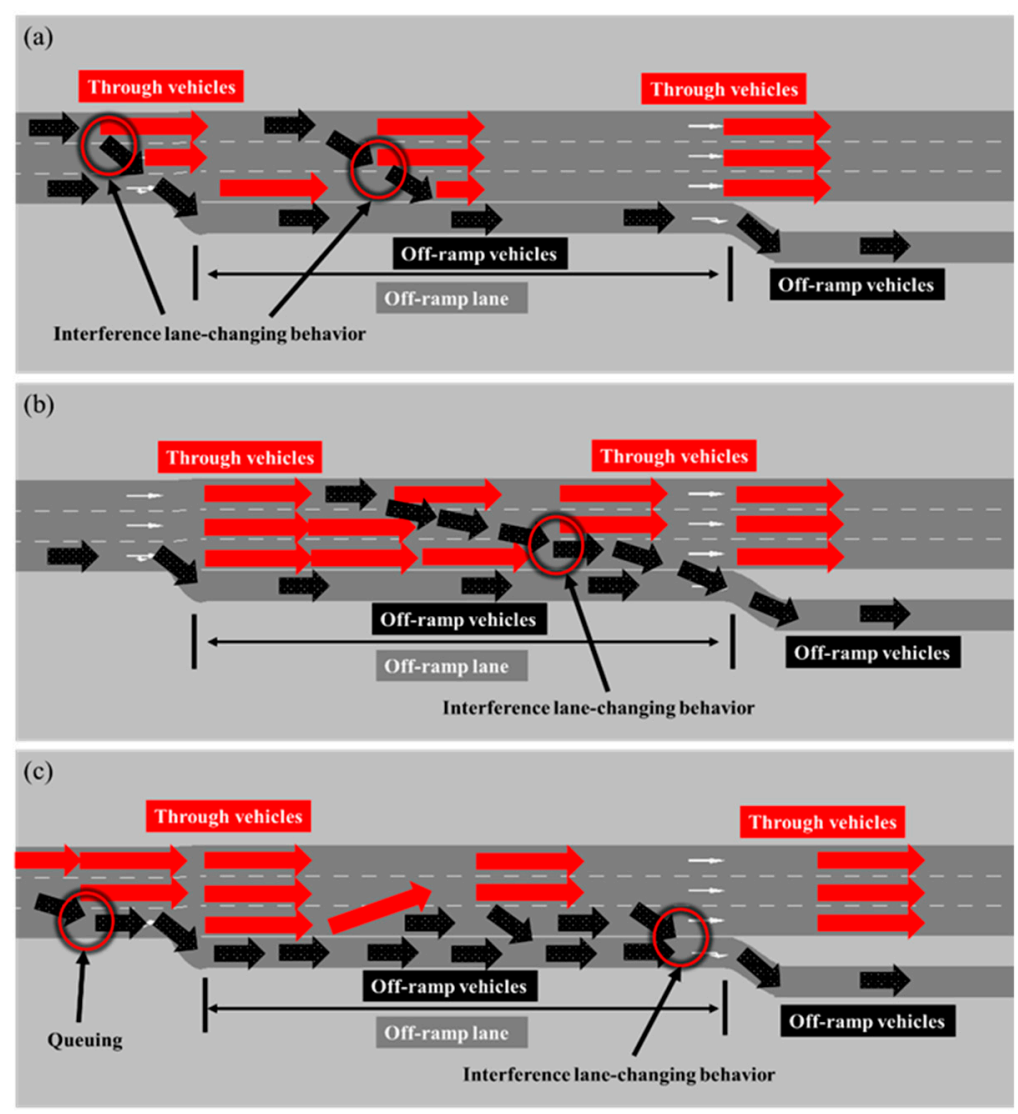

Figure 2. Lane-changing behaviors of off-ramp vehicles under different traffic conditions: (a) no traffic jam; (b) through traffic flow saturation or over-saturation; and (c) off-ramp traffic flow saturation or over-saturation.

\section{Data and Methodology}

\subsection{Investigation of Vehicle Lane-Changing Behavior in Off-Ramp Areas}

Two typical off-ramp areas in Beijing were investigated in this paper, which were off-ramp areas at the Yongdingmen bridge (YB) of 2nd-Ring Road and at the Taiyanggong bridge (TB) of 3rd-Ring Road. The off-ramp of YB connects the 2nd-Ring Road and the Dongbinhelu with a length of approximately $110 \mathrm{~m}$. The other off-ramp is approximately $500 \mathrm{~m}$ and connects the 3rd-Ring Road and the Jingcheng freeway.

Field investigation found that the YB segment is easily congested due to the heavy traffic volume of straight lanes. Some mainline vehicles take up off-ramp lanes, which lead to rear vehicle delays and cause some off-ramp vehicles to occupy the emergency lane. As shown in Figure 3a, vehicle A and vehicle $B$ are mainlined through vehicles, and they should change lanes to the straight lanes as early as possible instead of driving in the off-ramp lane, which causes the queuing phenomenon. Some off-ramp vehicles (such as vehicle $C$ and vehicle $D$ in Figure $3 a$ ) will drive in the emergency lane. If the through vehicles (such as vehicle A and vehicle B in Figure 3a) always drove in straight lanes 
before they got to the off-ramp area, the off-ramp lane may not queue, and the off-ramp vehicles $C$ and D may not drive in the emergency lane.
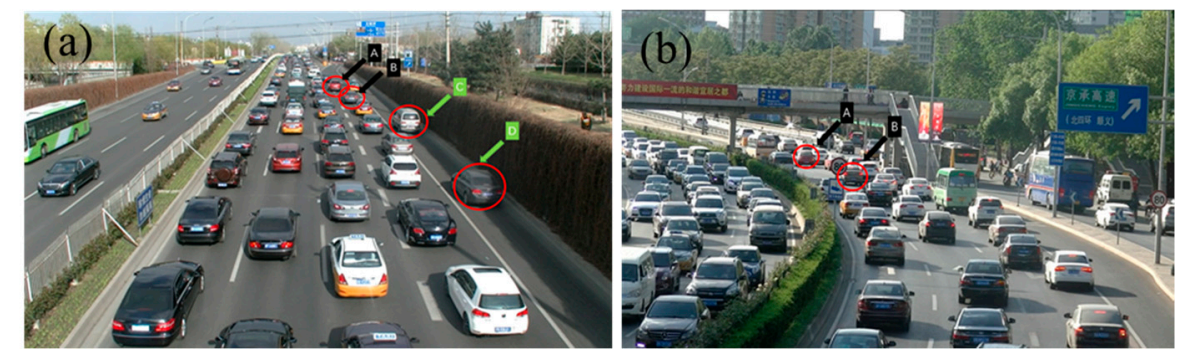

Figure 3. Lane-changing behavior of the field investigation in the off-ramp area of the Yongdingmen bridge (YB) and the Taiyanggong bridge (TB): (a) YB, and (b) TB.

Differing with $\mathrm{YB}$, the $\mathrm{TB}$ segment will be easily congested by off-ramp saturation. Some off-ramp vehicles do not want to queue in off-ramp lanes and take up straight lanes, which cause rear mainline vehicle delay. As shown in Figure 3b, the off-ramp vehicles (such as vehicle A and vehicle B in Figure $3 \mathrm{~b}$ ) should change lanes to the off-ramp lane as early as possible instead of driving in the straight lane and changing lanes right at the last minute, which delays the rear through vehicles. If the off-ramp vehicles can finish their lane-changing behaviors before they are very close to the off-ramp area, then the travel speed of straight lanes as well as the road capacity will be improved.

\subsection{Basic Simulation Scenario (Scenario I)}

In this paper, by means of the combination of theoretical analysis and traffic simulation, and by using the travel time and average speed obtained by VISSIM simulation as indicators, the appropriate lane-changing spacing of off-ramp areas was studied. This paper needed to analyze the individual behavior of vehicles under different traffic guidance ways. A VISSIM simulation can generate the average delay, average speed, travel time, and queue length of vehicles, and can describe driving behavior. At the same time, the built-in operation model of VISSIM is suitable for describing the lane-changing behavior of vehicles. Based on the research of this paper, the VISSIM simulation platform was selected for research. The VISSIM lane-changing model uses a rule-based lane-changing model. The time and spacing of lane-changing depend on the speed of the front vehicle and the vehicle wanting to change lanes. The lateral position of the vehicle has little influence on lane-changing behavior and lane-changing spacing. Therefore, the influence of the lateral position of the vehicle was not considered.

The basic simulation scenario was based on the field investigation of $Y B$ by setting traffic flow parameters (such as hourly volume and ratio of off-ramp vehicles) and basic road facility parameters (such as speed limit, ramp geometric dimensioning, and number of lanes) of the investigation area to simulate the traffic flow conditions of the off-ramp area. The simulation environment was VISSIM, and the lane-changing model adopted the free lane-changing model used in VISSIM. The length of simulation segment was $1800 \mathrm{~m}$ with an upstream part $1300 \mathrm{~m}$ away from the off-ramp exit section and a downstream part $500 \mathrm{~m}$ in length.

According to actual conditions of Scenario I, the upstream part of the YB segment away from the off-ramp exit section is a four-lane segment and the downstream part of the mainline segment is three lanes. The off-ramp is one lane and the side road connected to the off-ramp is two lanes.

Considering field traffic data and actual road geometric parameters, the volume unit was a standard passenger car, and the volume and speed limit parameters were as follows. The traffic volume of the straight lane was $1200 \mathrm{pcu} / \mathrm{h} / \mathrm{lane}$, and the corresponding speed limit was $80 \mathrm{~km} / \mathrm{h}$; the traffic volume of the side road was $400 \mathrm{pcu} / \mathrm{h} /$ lane, and the corresponding speed limit was $50 \mathrm{~km} / \mathrm{h}$; the ratio of off-ramp vehicles was $20 \% / \mathrm{h} /$ lane, and the speed limit of the off-ramp was $40 \mathrm{~km} / \mathrm{h}$. 
To insure the reliability and specificity of the simulation results and to study the impact of lane-changing spacing interval on traffic flow conditions at the off-ramp areas, the following assumptions were made to eliminate the influence of other factors: (1) there was no congestion of the mainline downstream, and (2) there was no congestion of the side road downstream.

\subsection{Lane-Changing Spacing Interval Parameters and Traffic Flow Detectors}

Let $\mathrm{S}$ denote the lane-changing spacing interval-a spacing interval where all vehicles (including mainline through vehicles and off-ramp vehicles) can make lane-changing behaviors freely to drive in their expected lane. Vehicles can change lanes according to their own wishes if the lane-change condition permits. The range of $S$ was from the section of free lane-changing to the separation point of ramp and mainline. Before the vehicles approached the section of free lane-changing, each vehicle drove in its initial lane, and the mainline through vehicles and the off-ramp vehicles were evenly distributed in each lane.

The average speed of each section and the travel time could effectively reflect the state of traffic flow. Two types of traffic detectors were set up in the simulation scenario: one was the traffic flow detector, which could collect the traffic volume and the average speed of the section in the statistics period (Detectors A and B); the other was the travel time detectors, which could detect the average travel time of the vehicles passing the given two sections in a statistical period (Detectors C, D1 and D2). Based on the simulation scenario, the position of the detector settings is shown in Figure 4. The traffic flow detection was set at $100 \mathrm{~m}$ upstream of the off-ramp section; the starting point of the travel time detector was set at $1200 \mathrm{~m}$ upstream of the off-ramp section, and two end points were separately set at the main road and the auxiliary road $300 \mathrm{~m}$ downstream of the off-ramp section.

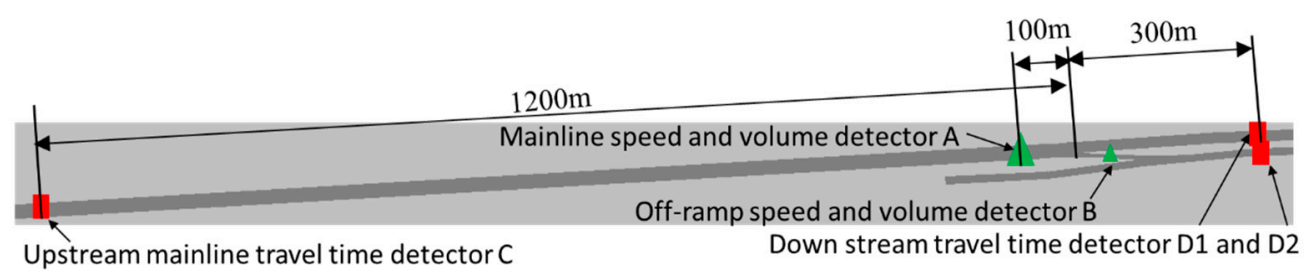

Figure 4. Distribution position of traffic detectors of the basic simulation scenario.

\subsection{Multi-Parameter Simulation Scenario (Scenario II)}

To study the relationship of lane-changing spacing interval and road capacity of the off-ramp, based on Scenario I, the relationship of lane-changing spacing interval and the state of traffic flow under different traffic volumes with a different ratio of off-ramp vehicles was studied. This simulation scenario can be regarded as Scenario II, whose basic simulation parameters were the same as Scenario I. The two indexes of sectional speed (SS) and travel time (TT) were selected to measure the traffic flow state.

\subsubsection{Traffic Volume Given Based Simulation Scenario (Scenario II.1):}

Let $\mathrm{S}$ and $\mathrm{r}$ be the lane-changing spacing interval vector and ratio of off-ramp vehicle of Scenario II.1. $S=[50,100, \ldots, 1200]$ (unit: $\mathrm{m}$ ), and $\mathrm{r}=[5 \%, 10 \%, \ldots, 50 \%]$. The given traffic volume was $1200 \mathrm{pcu} / \mathrm{h} /$ lane. The next step was to analyze the traffic flow state of Scenario II.1 with different lane-changing spacing intervals and ratios of off-ramp vehicles by different combinations of $S$ and $r$. The simulation period for each combination was one hour.

\subsubsection{Lane-changing Ratio Given Basic Simulation Scenario (Scenario II.2):}

Given $20 \%$ as the ratio of off-ramp vehicles, let $Q$ be the traffic flow volume for each lane and initialize $S=[50,100, \ldots, 1200]$ (unit: $\mathrm{m}$ ) and $\mathrm{Q}=[900,1000, \ldots, 1800]$ (unit: $\mathrm{pcu} / \mathrm{h} /$ lane) for Scenario 
II.2. The traffic flow state based on indexes SS and TT were studied in Scenario II.2 by different combinations of $S$ and $Q$. The simulation time was the same as Scenario II.1.

\section{Simulation Results and Discussion}

\subsection{Simulation Results of Scenario I}

Based on Scenario I, Figure 5 shows the traffic flow states of the off-ramp area for Scenario I at 30th min with different lane-changing spacing intervals. It can be seen intuitively in Figure 5 that the congestion states of traffic flow at the off-ramp area were very different with different lane-changing spacing intervals. The larger the lane-changing spacing interval was, the less congestion at the off-ramp area (or even no congestion). With a long enough lane-changing spacing interval, vehicles could pass through the off-ramp area quickly. With an increasing lane-changing spacing interval, vehicles could achieve non-interference lane-changing behaviors as soon as possible. The off-ramp vehicles could change to outer lanes without interference as early as possible and provide more space to other vehicles so that the mainline through vehicles could change to inner lanes in advance. Then, at off-ramp areas, a kind of diverging flow could be formed, which decreased the probability of congestion. In contrast, when the lane-changing spacing interval was insufficient, the off-ramp vehicles would change from inner lanes to outer lanes in a limited spacing interval, which led to interference lane-changing behaviors. Some vehicles may decelerate or even stop in inner lanes, delaying rear mainline through vehicles. These vehicles occupied inner lanes and greatly affected non off-ramp vehicles in changing to inner lanes. Then, interference lane-changing behaviors occurred in both mainline through vehicles and off-ramp vehicles, which led to congested weaving flow and easily formed local congestion. As time passes by, the congestion further spreads to upstream segments. Finally, it could cause regional congestion, which seriously reduces the road capacity of the off-ramp area.

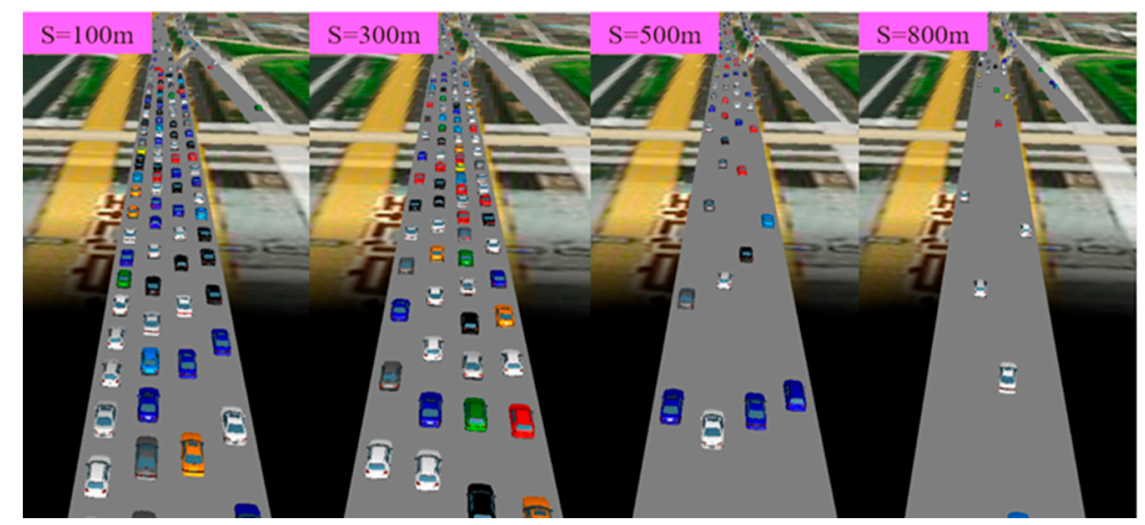

Figure 5. Traffic flow states of off-ramp area for Scenario I at 30th min with different lane-changing spacing intervals.

Table 1 shows the traffic volume results of detectors A (upstream mainline volume) and B (off-ramp volume) (see Figure 4) under different lane-changing spacing intervals for a one-hour simulation in Scenario I. With an increasing lane-changing spacing interval, the corresponding traffic volume increased. When the lane-changing spacing interval was larger than $400 \mathrm{~m}$, the current traffic facilities could fit the requirements of traffic demands (as shown in Scenario I), which were 4800 pcu (the second column in Table 1) in total (four lanes) and 960 pcu (the third column in Table 1) in the on-ramp ( $20 \%$ of off-ramp vehicles). Because some vehicles upstream did not yet pass detectors A and $\mathrm{B}$ when the simulation was done, the outputs of detectors $\mathrm{A}$ and $\mathrm{B}$ were a little bit smaller than vehicles that passed detector $\mathrm{C}$. 
Table 1. Outputs of detectors A and B of Scenario I.

\begin{tabular}{ccc}
\hline $\begin{array}{c}\text { Lane-Changing } \\
\text { Spacing Interval (m) }\end{array}$ & $\begin{array}{c}\text { Outputs of } \\
\text { Detector A }(\# \mathbf{p c u} / \mathbf{h})\end{array}$ & $\begin{array}{c}\text { Outputs of } \\
\text { Detector B (\#pcu/h) }\end{array}$ \\
\hline 50 & 2581 & 488 \\
100 & 2895 & 542 \\
150 & 3086 & 591 \\
200 & 3325 & 654 \\
250 & 3595 & 716 \\
300 & 4017 & 791 \\
350 & 4017 & 798 \\
400 & 4732 & 928 \\
450 & 4732 & 929 \\
500 & 4732 & 928 \\
550 & 4732 & 930 \\
600 & 4733 & 930 \\
650 & 4733 & 929 \\
700 & 4734 & 930 \\
750 & 4733 & 928 \\
800 & 4733 & 930 \\
850 & 4733 & 930 \\
900 & 4733 & 930 \\
950 & 4734 & 930 \\
1000 & 4733 & 930 \\
1050 & 4732 & 930 \\
1100 & 4732 & 930 \\
1150 & 4732 & 931 \\
1200 & 4730 & 923 \\
\hline
\end{tabular}

\subsubsection{Analyzing Sectional Speed Index (SSI):}

Figure 6 shows SSI with different lane-changing spacing intervals. When the lane-changing spacing was larger than $400 \mathrm{~m}$, the vehicles basically had their ideal driving speeds, which could guarantee the design capacity at the off-ramp area. When the lane-changing spacing was less than $400 \mathrm{~m}$, congestion occurred and the vehicles moved slowly. The less the lane-changing spacing was, the more serious the congestion. When the lane-changing spacing increased from 350 to $400 \mathrm{~m}$, the corresponding speed had a distinct jump, which meant congestion could occur in a qualitative manner. Sometimes, a small disturbance (such as a random vehicle arriving) will lead to a tiny congestion. If the tiny congestion cannot be released in time, it will aggravate rapidly and spread upstream.

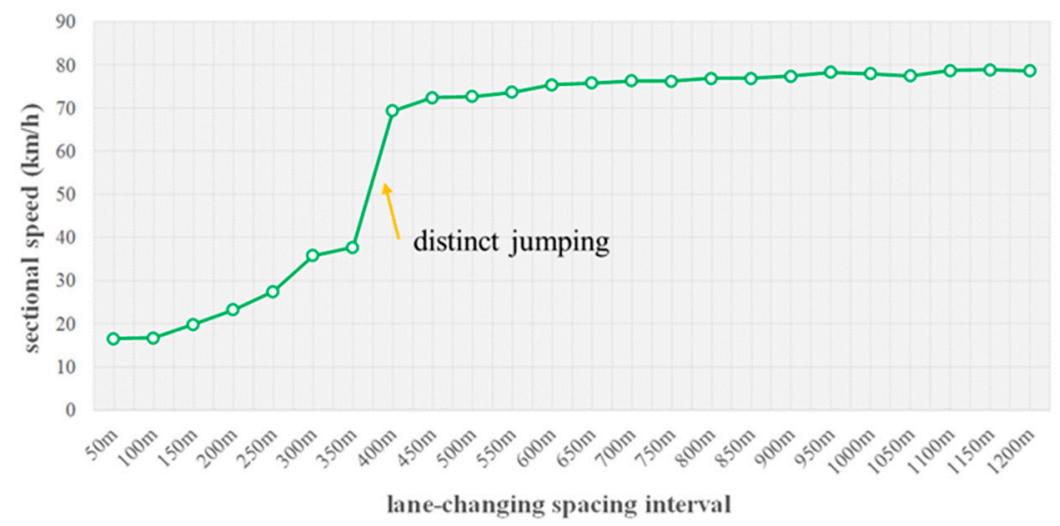

Figure 6. Sectional speed index (SSI) based on Detector A under different lane-changing spacing intervals. 


\subsubsection{Analyzing Travel Time Index (TTI):}

TTI can also reflect the traffic state of the off-ramp area. Figure 7 shows TTIs with different lane-changing spacing intervals. When the lane-changing spacing interval was large, it means no congestion will happen in the ramp area, then the travel time at the off-ramp area was short. When the lane-changing spacing interval was small, the speed was low and the travel time was long. A long travel time meant that the traffic flow was in a congested state and the vehicles moved slowly. Similar with Figure 6, a distinct jump of TTI between 350 and $400 \mathrm{~m}$ can also be observed in Figure 7.

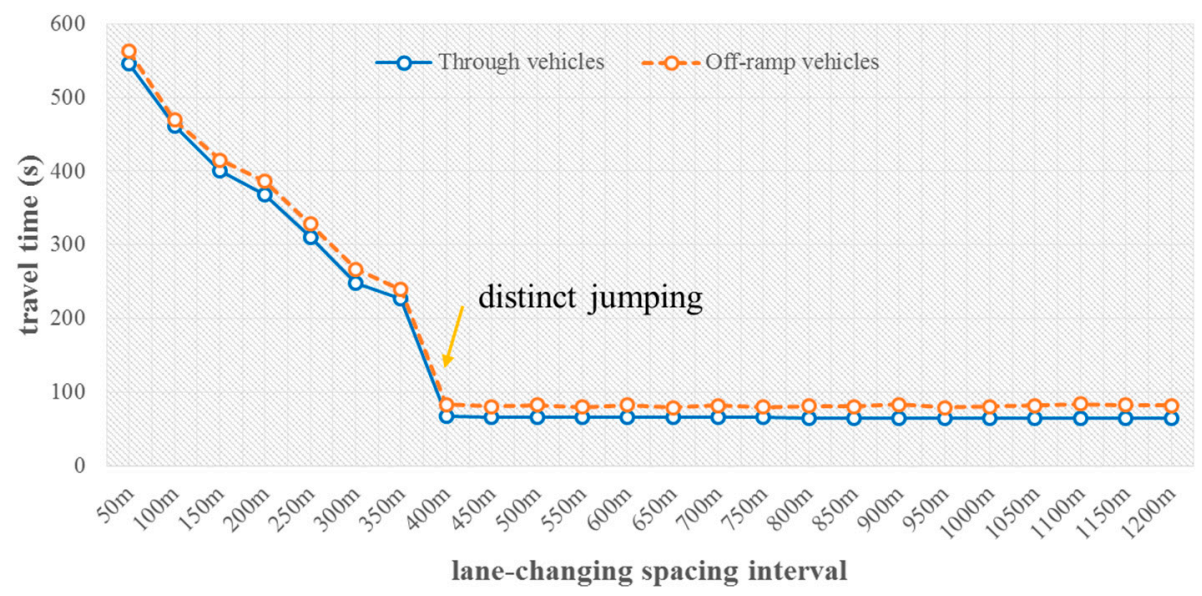

Figure 7. Travel time indexes (TTIs) between sections of Detector C and sections of Detectors D1 and D2.

\subsection{Simulation Results of Scenario II}

\subsubsection{Analyzing SSI:}

It can be seen from Figure 8a, for the same traffic volume, the larger the lane-changing spacing interval was, the higher the SSI was. For the same lane-changing space, the smaller the traffic volume was, the higher the SSI was. In no congestion conditions the value of SSI eventually tended towards the expected speed. When the traffic volume was approximately $1200 \mathrm{pcu} / \mathrm{h} /$ lane, the lane-changing spacing interval for these vehicles was not less than $500 \mathrm{~m}$. When the traffic volume was less than $1000 \mathrm{pcu} / \mathrm{h} /$ lane, the spacing interval $300 \mathrm{~m}$ was enough for these vehicles to reach their expected speeds. When the traffic volume was from 1200 to $1500 \mathrm{pcu} / \mathrm{h} /$ lane, the desired lane-changing spacing interval was from 500 to $1200 \mathrm{~m}$. When the traffic volume was more than $1500 \mathrm{pcu} / \mathrm{h} / \mathrm{lane}$, the spacing interval $1200 \mathrm{~m}$ did not fit the requirement for these vehicles. At this point, there was many interference lane-changing behaviors, which easily caused traffic congestion. Then, a large enough lane-changing spacing interval will be necessary for heavy traffic volume.

For Figure $8 \mathrm{~b}$, given the ratio of off-ramp vehicles, the larger the lane-changing spacing interval was, the higher the SSI was. For the same lane-changing spacing interval, a larger ratio of off-ramp vehicles led to a lower SSI. To have smooth traffic flow, a larger ratio of off-ramp vehicles required a larger lane-changing spacing interval. As shown in Figure 8b, when the ratio of off-ramp vehicles was less than $25 \%$, a $500 \mathrm{~m}$-long lane-changing spacing interval guaranteed ideal speeds for these vehicles. When the ratio of the off-ramp vehicles was between $25 \%$ and $40 \%, 500$ to $1000 \mathrm{~m}$ was needed for lane-changing spacing. In addition, when the ratio was more than $40 \%$, vehicles could not always reach their ideal driving speed for a lane-changing spacing interval of $1200 \mathrm{~m}$. At this point, the relationship of the lane-changing spacing interval and SSI was a positive correlation. In some key off-ramp areas, through traffic management and guidance to reduce the ratio of off-ramp vehicles, the congestion could be relieved effectively. A kind of active traffic management-smart ramp management-could be implemented by using adjacent multi-ramps to share peak hour traffic volume. 

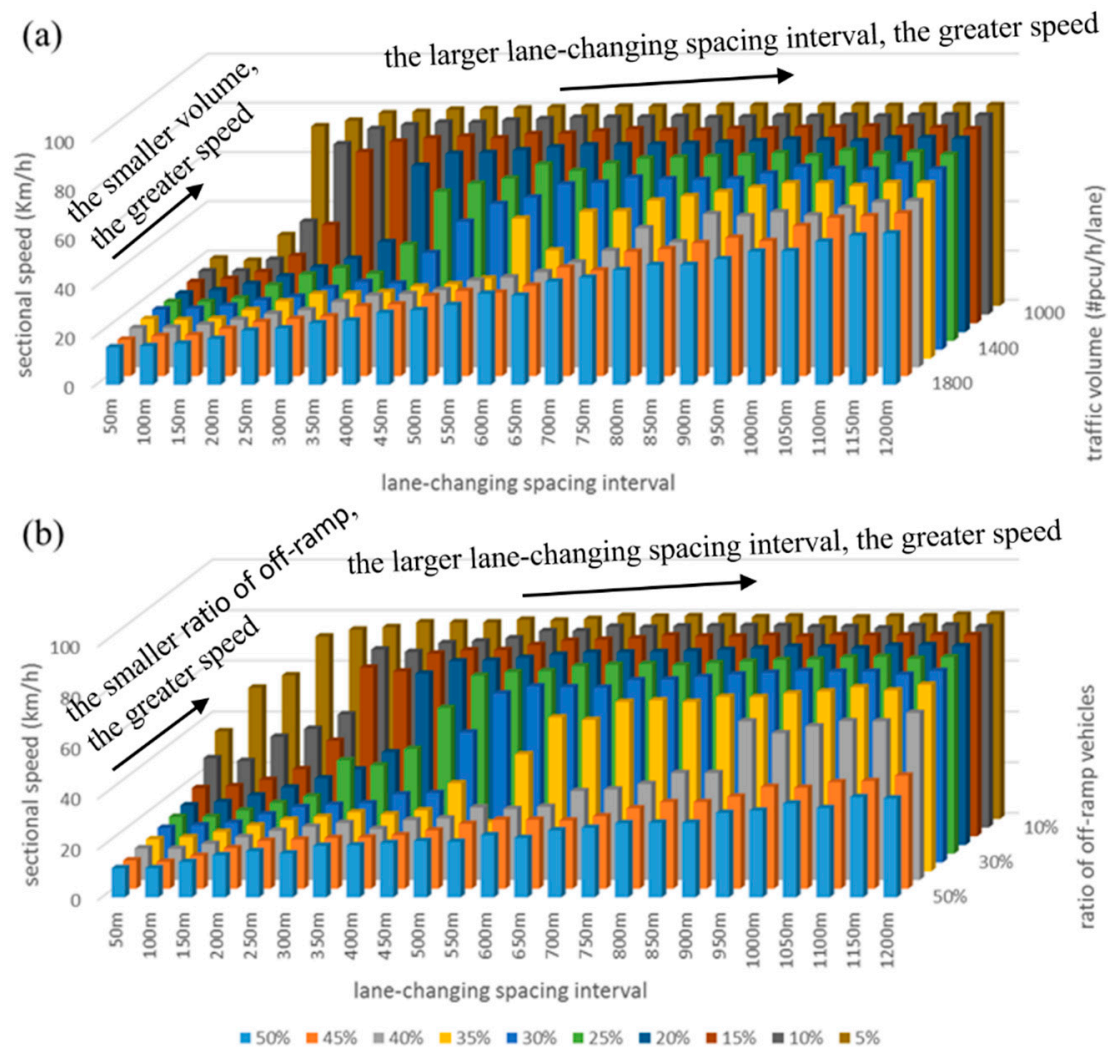

Figure 8. Three-dimensional state diagrams of Scenario II based on SSI: (a) Scenario II.1; and (b) Scenario II.2.

\subsubsection{Analyzing TTI:}

Figure 9 shows the three-dimensional state diagrams of Scenario II based on TTI. As shown in Figure $9 a, b$, for the same traffic volume, the larger the lane-changing spacing interval was, the shorter the travel time will be. A larger lane-changing spacing interval could disperse the negative impact of lane-changing behavior. When the traffic volume increased, a larger lane-changing spacing interval would be required. When the traffic volume was $900 \mathrm{pcu} / \mathrm{h} /$ lane, the corresponding lane-changing spacing interval was $200 \mathrm{~m}$, which could ensure a short travel time. Then, for every $100 \mathrm{pcu} / \mathrm{h} / \mathrm{lane}$ of traffic volume increase, the corresponding lane-changing spacing interval increased by approximately $50 \mathrm{~m}$ to ensure a non-congestion traffic state at off-ramp areas.

Figure $9 c, d$ are the three-dimensional state diagrams of mainline through vehicles and off-ramp vehicles based on TTI with different ratios of off-ramp vehicles. For the same ratio, with an increase in the lane-changing spacing interval, TTI decreased to a fixed value, which referred to a free flow state. It was recommended that the off-ramp vehicles should change to outer lanes as early as possible to avoid aggravating congestion. For a larger ratio of off-ramp vehicles, the lane-changing spacing interval should have enough lane-changing distance and meet lane-changing demand between these vehicles. When the ratio of off-ramp vehicles was less than $40 \%$, the TTI decreased distinctly with an increase in the lane-changing spacing interval. When the ratio was over $40 \%$, the value of lane-changing spacing interval had less influence on TTI because the traffic in the off-ramp area had to be in a congested state. 

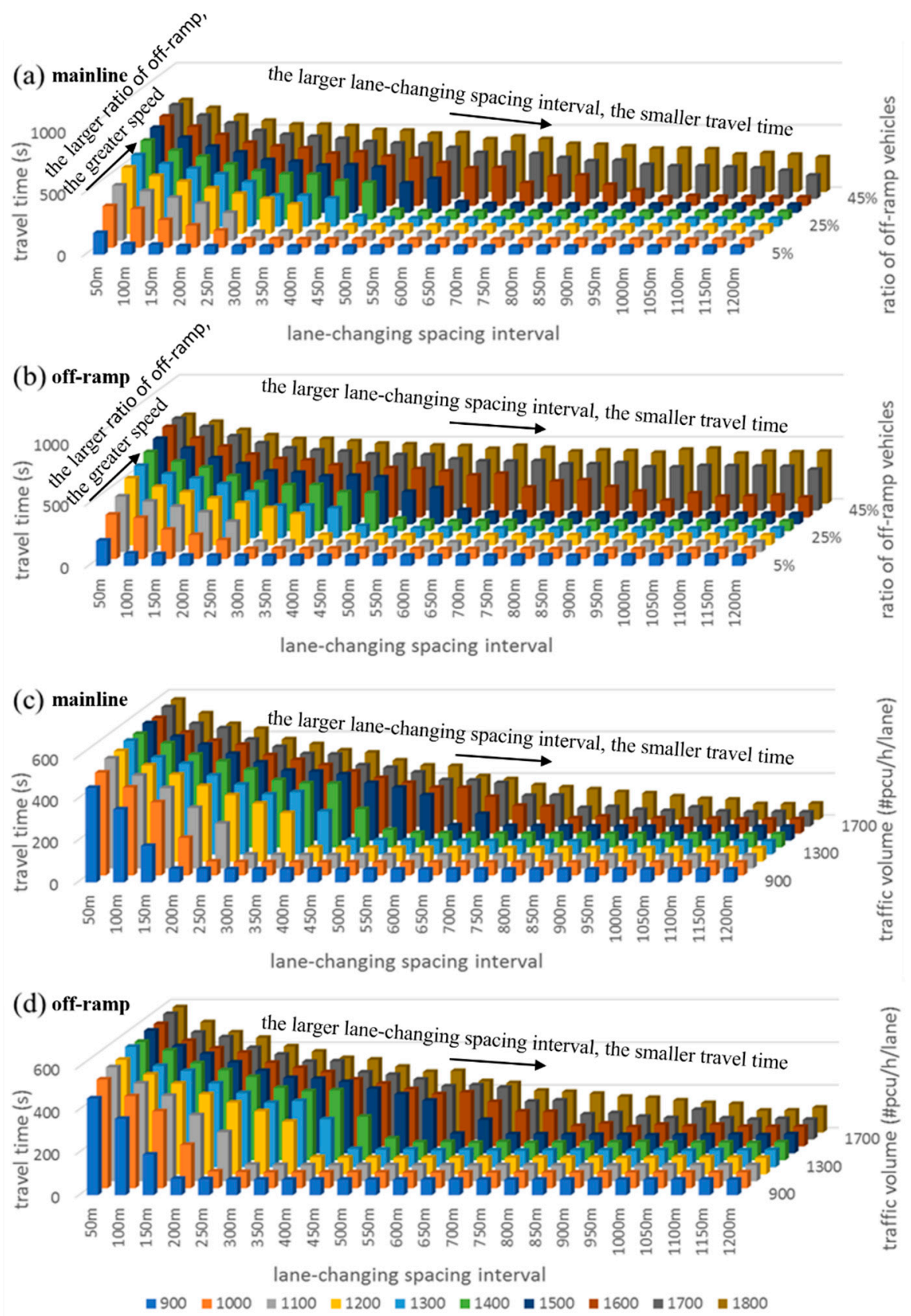

Figure 9. Three-dimensional state diagrams of Scenario II based on TTI: (a) Scenario II.1 between Detector C and Detector D1; (b) Scenario II.1 between Detector C and Detector D2; (c) Scenario II.2 between Detector C and Detector D1; and (d) Scenario II.2 between Detector C and Detector D2.

\subsection{Discussion}

Based on the actual investigation data, this paper used VISSIM to simulate various scenarios, such as different lane-changing spacing intervals, different traffic volumes, and different on-ramp ratios, and then compared the results. VISSIM simulation is widely used in research, and its output results and methods have sufficient accuracy and credibility. However, in this paper, research on the appropriate lane-changing spacing for off-ramp areas of urban expressways was only theoretical and remained in the VISSIM simulation; it has not been verified in practice. Because the study of lane-changing spacing in this paper is relatively novel, it has not been applied in expressways. For guiding practical applications based on the method proposed in this paper, Figure 10 shows a flow chart to determine appropriate lane-changing spacing intervals for general cases. In order to 
make the conclusions more convincing, further research will progress with the development of driving simulation scenarios and the recruitment of drivers for experiments.

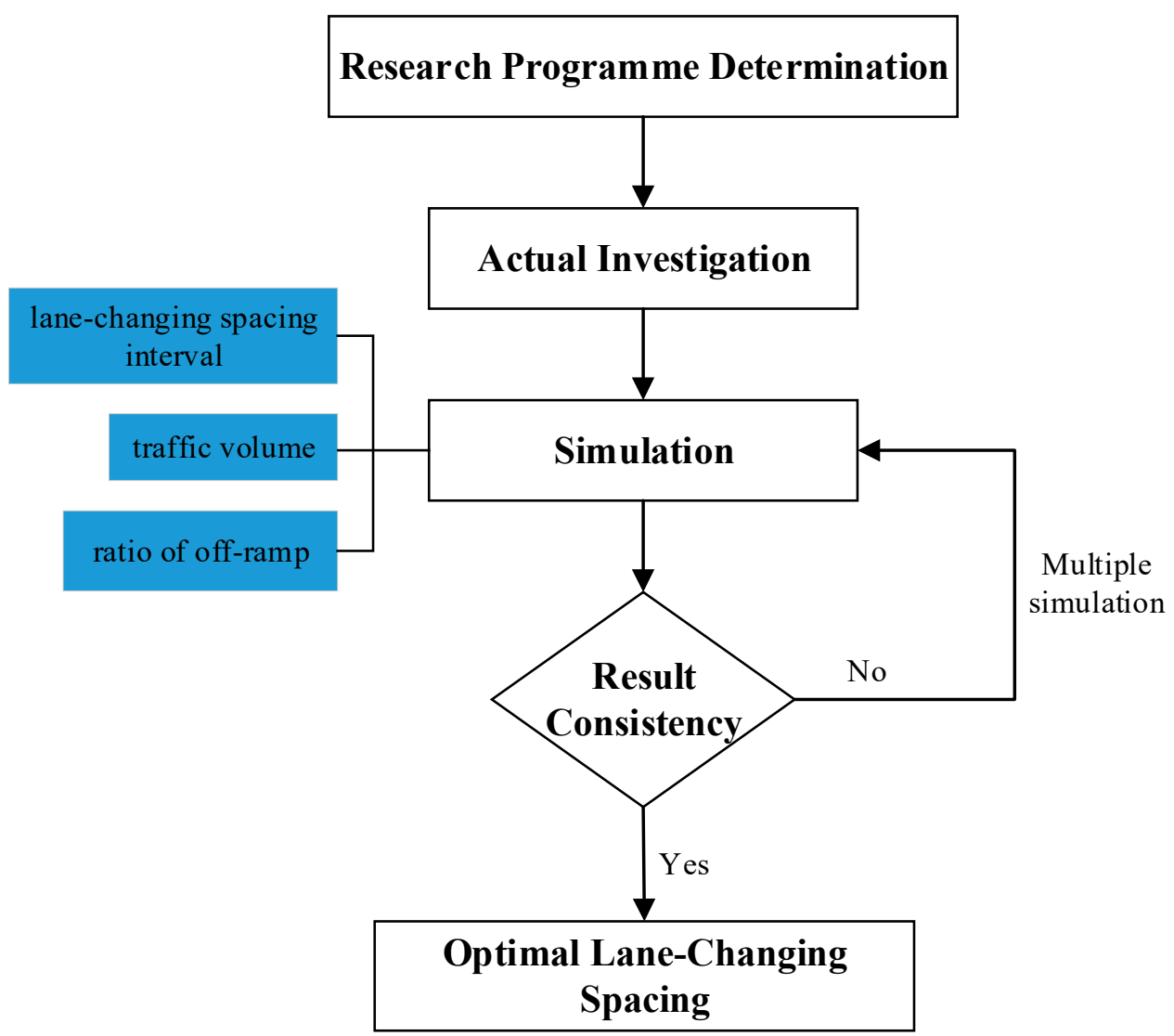

Figure 10. A flow chart to determine appropriate lane-changing spacing intervals for general cases.

\section{Conclusions}

Based on the actual investigation data, the driving behaviors in off-ramp areas of urban expressways are analyzed and simulated to study the relationship of a lane-changing spacing interval and traffic flow state with different traffic volumes and different ratios of off-ramp vehicles. Insufficient lane-changing spacing intervals for certain traffic volume demand causes congestion, because the off-ramp vehicles will always change lanes intensively when they travel to the off-ramp area. These behaviors will affect the through vehicles and lead to unnecessary delays for rear vehicles. In engineering, one way to avoid unnecessary delays is to determine reasonable lane-changing spacing intervals for certain off-ramp areas and traffic volume demands. Another way is to avoid compressing existing lane-changing spacing intervals by guiding the lane-changing behavior of the vehicles.

Therefore, an appropriate lane-changing spacing interval is the basic guarantee for road safety and road capacity. Bad driving behaviors will lead to congestion. With a sufficient lane-changing spacing interval, traffic managers need to guide drivers to make full use of existing lane-changing spacing intervals effectively to avoid interference lane-changing behaviors. If off-ramp vehicles always change lanes just near the exit of an off-ramp, especially some off-ramp vehicles driving in the inner lanes, they will severely disturb the normal driving of through vehicles and cause unnecessary delay for rear vehicles. These behaviors will disturb traffic flow and probably lead to congestion or traffic accidents. Thus, it is necessary to standardize lane-changing behaviors, especially for off-ramp vehicles, to reduce interference lane-change behavior. This can improve the smoothness of traffic flow and help to avoid traffic accidents caused by compulsive lane-changing behaviors. A kind of "smart lane management" can be adopted in future traffic management. A combination strategy by using fixed 
traffic signs, traffic markings, and various message signs can be implemented to standardize and restrain lane-changing behaviors in off-ramp areas.

The results of this paper are useful in several aspects: (1) to further optimize lane-changing behaviors to improve road capacity; (2) to provide a reference for location selection and design of urban expressway entrances and exits; and (3) to guide the design for an appropriate combination of strategies of lane-changing behavior guidance. In future studies we can focus on the following: (1) lane-changing behaviors and active lane management strategies in weaving and diverging areas; and (2) appropriate constraint methods of lane-changing behavior with different traffic flow states and their corresponding engineering designs for lane-changing behavior guidance and constraint.

Author Contributions: Conceptualization, H.L. and J.R.; methodology, Z.H. and Z.Z.; investigation, Z.H. and Z.Z.; writing - original draft preparation, H.L., and L.Q.; project administration, H.L.; All authors read, revised and approved the final version of the original manuscript. Moreover, several native English speakers contributed to paper revision before submission.

Funding: This work is supported by the Natural Science Foundation (51608017) and the International Research Cooperation Seed Fund of Beijing University of Technology (No. 2018B21).

Conflicts of Interest: The authors declare no conflict of interest.

\section{References}

1. AASHTO (American Association of State Highway and Transportation Officials). HIGHWAY SAFETY MANUAL; American Association of State Highway and Transportation Officials: Washington, DC, USA, 2010; ISBN 9781560514770.

2. Qi, L.; Member, S.; Zhou, M.; Luan, W.; Member, S. Impact of Driving Behavior on Traffic Delay at a Congested Signalized Intersection. IEEE Trans. Intell. Transp. Syst. 1882, 18, 1882-1893. [CrossRef]

3. Cao, X.; Young, W.; Sarvi, M.; Kim, I. A study of mandatory lane-changing execution behaviour model for heavy. Australas. Transp. Res. Forum 2016, 38, 1-18.

4. Jin, W.-L. A multi-commodity Lighthill-Whitham-Richards model of lane-changing traffic flow. Procedia-Soc. Behav. Sci. 2013, 80, 658-677. [CrossRef]

5. Kanaris, A.; Kosmatopoulos, E.B.; Ioannou, P.A. Strategies and spacing requirements for lane changing and merging in automated highway systems. IEEE Trans. Veh. Technol. 2001, 50, 1568-1581. [CrossRef]

6. Liu, X.-H.; Ko, H.-T.; Guo, M.-M.; Wu, Z. A new traffic model on compulsive lane-changing caused by off-ramp. Chin. Phys. B 2016, 25. [CrossRef]

7. Wan, X.; Jin, P.; Yang, F.; Zhang, J.; Ran, B. Modeling vehicle interactions during merge in congested weaving section of freeway ramp. Transp. Res. Rec. J. Transp. Res. Board 2014, 2421, 82-92. [CrossRef]

8. Keyvan-Ekbatani, M.; Knoop, V.L.; Daamen, W. Categorization of the lane change decision process on freeways. Transp. Res. Part C Emerg. Technol. 2016, 69, 515-526. [CrossRef]

9. Keyvan-Ekbatani, M.; Knoop, V.L.; Grébert, V.; Daamen, W. Lane change strategies on freeways: A microscopic simulation study. In Traffic Granul. Flow '15; Springer: Cham, Switzerland, 2015; pp. 395-402. [CrossRef]

10. Khan, U.; Basaras, P.; Schmidt-Thieme, L.; Nanopoulos, A.; Katsaros, D. Analyzing cooperative lane change models for connected vehicles. In Proceedings of the 2014 International Conference on Connected Vehicles and Expo (ICCVE), Vienna, Austria, 3-7 November 2014; pp. 565-570. [CrossRef]

11. Wang, X.Y.; Wang, J.Q.; Zhang, J.L.; Ban, X.G. Lane-changing model with dynamic consideration of driver's propensity. Int. J. Mod. Phys. C 2015, 26, 19. [CrossRef]

12. Li, H.; Li, S.; Li, H.; Qin, L.; Li, S.; Zhang, Z. Modeling Left-Turn Driving Behavior at Signalized Intersections with Mixed Traffic Conditions. Math. Probl. Eng. 2016, 2016, 4015271. [CrossRef]

13. Balal, E.; Cheu, R.; Gyan-Sarkodie, T.; Miramontes, J. Analysis of Discretionary Lane Changing Parameters on Freeways. Int. J. Transp. Sci. Technol. 2014, 3, 277-296. [CrossRef]

14. Du, Y.; Wu, G.; Boriboonsomsin, K.; Chan, C.-Y. Empirical Study of Lane-Changing Behavior Along Different Types of High-Occupancy Vehicle Facilities in California. Transp. Res. Rec. J. Transp. Res. Board 2013, 2396, 143-150. [CrossRef] 
15. Hill, C.; Elefteriadou, L.; Kondyli, A. Exploratory analysis of lane changing on freeways based on driver behavior. J. Transp. Eng. 2006, 141, 1-11. [CrossRef]

16. Hou, Y.; Edara, P.; Sun, C. Modeling mandatory lane changing using bayes classifier and decision trees. IEEE Trans. Intell. Transp. Syst. 2014, 15, 647-655. [CrossRef]

17. Zeng, J.; Yu, S.; Qian, Y.; Feng, X. Expressway traffic flow model study based on different traffic rules. IEEE/CAA J. Autom. Sin. 2018, 5, 1099-1103. [CrossRef]

18. Wang, F.; Zheng, N.; Cao, D.; Martinez, C.M.; Li, L.; Liu, T. Parallel Driving in CPSS: A Unified Approach for Transport Automation and Vehicle Intelligence. IEEE/CAA J. Autom. Sin. 2017, 4, 577-587. [CrossRef]

19. Mai, T.; Jiang, R.; Chung, E. A Cooperative Intelligent Transport Systems (C-ITS)-based lane-changing advisory for weaving sections. J. Adv. Transp. 2016, 50, 752-768. [CrossRef]

20. Tang, T.Q.; Wang, Y.P.; Yang, X.B.; Huang, H.J. A Multilane Traffic Flow Model Accounting for Lane Width, Lane-Changing and the Number of Lanes. Netw. Spat. Econ. 2014, 14, 465-483. [CrossRef]

21. Gong, S.; Du, L. Optimal location of advance warning for mandatory lane change near a two-lane highway off-ramp. Transp. Res. Part B Methodol. 2016, 84, 1-30. [CrossRef]

22. Seraj, M. Variable Speed Limit Compliance Impact on Bottleneck Lane Changing Pattern. Master's Thesis, University of Alberta, Edmonton, AB, Canada, June 2016.

23. Yun, M.; Zhao, J.; Zhao, J.; Weng, X.; Yang, X. Impact of in-vehicle navigation information on lane-change behavior in urban expressway diverge segments. Accid. Anal. Prev. 2017, 106, 53-66. [CrossRef]

24. Marczak, F.; Daamen, W.; Buisson, C. Key Variables of Merging Behaviour: Empirical Comparison between Two Sites and Assessment of Gap Acceptance Theory Key variables of merging behaviour: Empirical comparison between two sites and assessment of gap acceptance theory. Procedia-Soc. Behav. Sci. 2013, 80, 678-697. [CrossRef]

25. Leclercq, L.; Chiabaut, N.; Laval, J.; Buisson, C. Relaxation Phenomenon After Lane Changing Experimental Validation with NGSIM Data. Transp. Res. Record 1999, 79-85. Available online: https:/ / www.researchgate.net/publication/237137593_Relaxation_phenomenon_after_changing_lanes_ an_experimental_validation_with_the_NGSIM_dataset (accessed on 24 March 2019). [CrossRef]

26. Knoop, V.L.; Buisson, C.; Arem, B.V. Lane distribution of traffic near merging zones influence of variable speed limits. In Proceedings of the 13th International IEEE Conference on Intelligent Transportation Systems, Funchal, Portugal, 19-22 September 2010. [CrossRef]

27. Laval, J.A.; Daganzo, C.F. Lane-changing in traffic streams. Transp. Res. Part B Methodol. 2006, 40, $251-264$. [CrossRef]

28. Zheng, Z. Recent developments and research needs in modeling lane changing. Transp. Res. Part B Methodol. 2014, 60, 16-32. [CrossRef]

(c) 2019 by the authors. Licensee MDPI, Basel, Switzerland. This article is an open access article distributed under the terms and conditions of the Creative Commons Attribution (CC BY) license (http://creativecommons.org/licenses/by/4.0/). 